\title{
A Novel Energy Efficient Mobility Aware MAC Protocol for Wireless Sensor Networks
}

\author{
Zain ul Abidin Jaffri, Asif Kabir \\ College of Communication Engineering \\ Chongqing University \\ Chongqing 400044, China
}

\author{
Gohar Rehman Chughtai, S. Sabahat H. Bukhari \\ College of Computer Science \\ Chongqing University \\ Chongqing 400044, China
}

\author{
Muhammad Arshad Shehzad Hassan \\ College of Electrical Engineering \\ Chongqing University \\ Chongqing 400044, China
}

\begin{abstract}
Dealing with mobility at the link layer in an efficient and effective way is a formidable challenge in Wireless Sensor Networks due to recent boom in mobile applications and complex network scenarios. Most of the current MAC protocols proposed for WSNs generally focus on stationary network and usually provide feeble network performance in situations where mobile nodes are involved. Many MAC protocols are proposed and techniques are developed to support mobility but they undergo massive energy consumption and latency problems due to frequent connection setup and breakup. In this paper, we propose a new energy efficient mobility aware based MAC protocol (EEMA-MAC), which work efficiently in both stationary and mobile scenarios with less energy consumption. In this protocol the member nodes have sleep and awake time same like existing S-MAC protocol but it expedite the connection setup and efficiency as Cluster Head (CH) has extended wake up time and less sleep time. Simulation results show that this mechanism is effective to avoid frequent disconnection of nodes and performs well in terms of energy consumption, throughput and packet loss as compared with existing protocols, such as S-MAC and MS-MAC.
\end{abstract}

Keywords-Wireless sensor networks; energy efficiency; Media Access Control (MAC); mobility aware; cluster head

\section{INTRODUCTION}

Wireless Sensor Networks (WSNs) have major contribution in the recent boom of technological advancements. This technology has emerged with high potential to sense physical phenomena like environmental monitoring, medical systems, seismic events, smart spaces, etc. [1] by initially processing the collected data locally and then delivering this data over a multihop link [4]. Basically the network comprises of numerous distributed nodes which self-organize themselves as a wireless multi-hop network. Each node is battery operated and has one or more sensors, low power radios and embedded processors [1], [10]. Typically the nodes establish and maintain the network and coordinate to accomplish the assigned task. The network offers many attractive features due to their small size and can be easily deployed to the inaccessible places and the areas which are expensive for wired systems. Wireless Sensor Networks have large number of applications. They are used for health monitoring, fire and smoke, temperature, vibrations etc. Similar applications include structural health monitoring using accelerometer sensors [6], observing the activities of sea birds by using light, barometric, temperature and humidity sensors [5], to monitor active volcanos using infrasonic and seismic sensors [7] and to examine large water transmission pipelines using acoustic and hydraulic sensors [8].

The main focus of most of the applications above is on the nodes which are generally static after the deployment is done. In recent years mobility has emerged as a major constituent of many WSN applications [12]. The concept of mobility in WSNs is that few network elements like base station, sensor nodes, actuators or monitored targets can be mobile to enhance the capabilities of the system so that it can react quickly in many emergency situations [11]. As gradually the quantity of mobile units monitored by sensors are increasing, the role of mobility is also becoming highly important in WSNs. Most of the medium access control protocol designed recently are adopted for stationary networks. In these stationary networks the topology is fixed and the neighboring nodes remain unmoved for long period of time. As per the survey, only few MAC protocols support mobility, which creates room for research regarding mobility in WSNs [2], [3]. The MAC protocols can be divided into three types; Time division Multiple Access based (TDMA), contention based, and hybrid. In our current research we will focus on the contention-based MAC protocols. The contention-based protocols are less complicated in terms of using scheduling algorithm for channel division and maintain equal probability to access the channel in terms of both static and mobile nodes. In addition to this they do not encounter the overhead produced by hybrid protocols.

The main objective of our novel MAC protocol for is to work effectively and efficiently in both mobile and stationery scenarios with minimum energy consumption. In order to achieve our objective, S-MAC [1], a Sensor Medium Access control protocol is our first milestone and a starting point and then extends the protocol to support mobile sensors. The mobility-aware Sensor MAC protocol (MS-MAC) [9] works almost similar to the S-MAC for energy conservation when the nodes are stationary. 


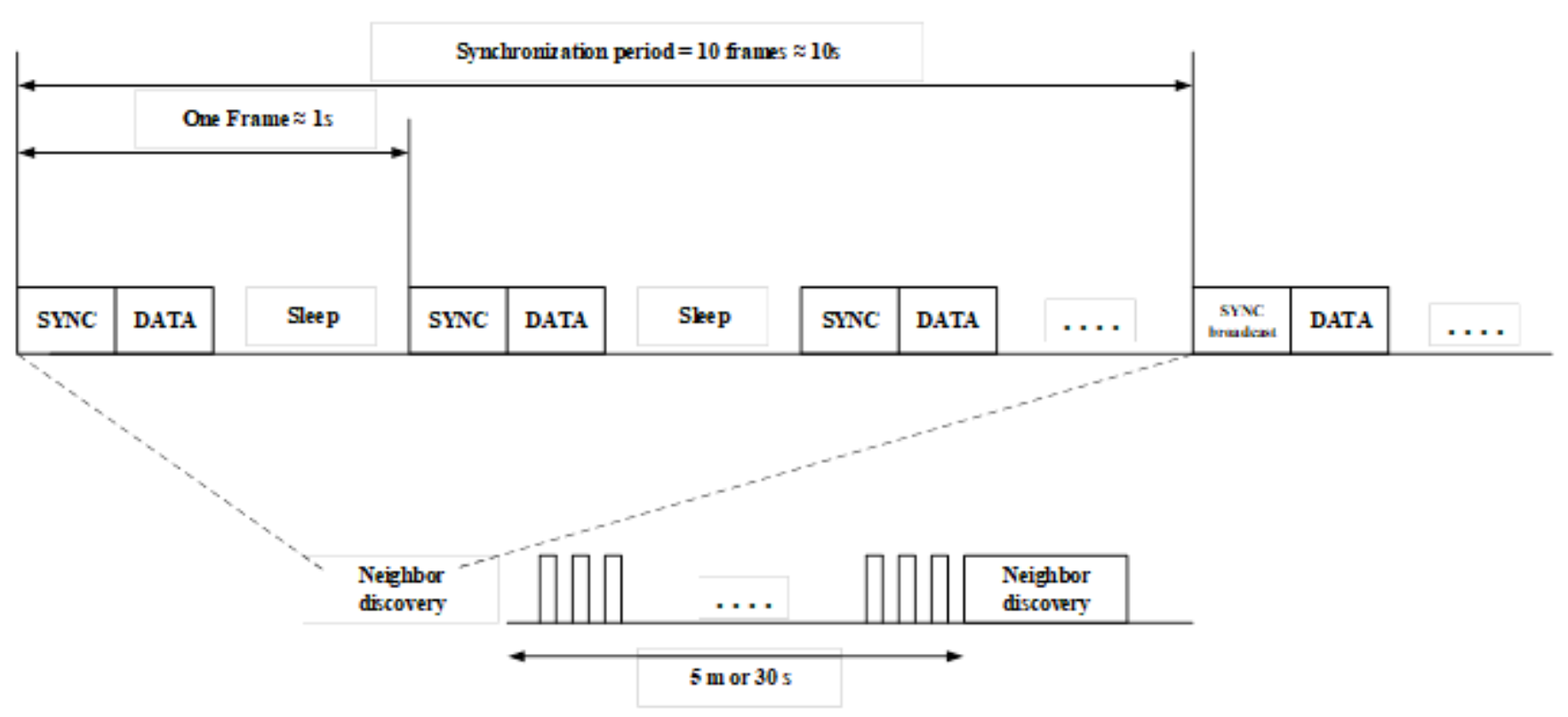

Fig. 1. Example S-MAC synchronization period description.

This medium access scheme can also switch to function like IEEE 802.11 for mobile ad-hoc scenario. S-MAC is basically a CSMA/CA MAC protocol, based on IEEE 802.11 which initiates periodic coordinated sleep/wakeup duty cycles as in Fig. 1, thus enhancing the lifetime of sensor nodes. To retain synchronization, for all predefined number of cycles, every node broadcasts its schedule in a SYNC message, so that its neighbors can update that information in their schedule tables accordingly. In addition to this, every node follow periodically the neighbor discovery scheme, so that the two neighbor nodes couldn't see each other (for example, because of SYNC packet corruption, interference, or because the medium remain busy and SYNC packets cannot be transmitted in time). The synchronization period (10 seconds) is repeated after every 2 minutes for this scheme. S-MAC does not need all the nodes in whole network, but only in each virtual cluster to synchronize. Border nodes amongst virtual clusters are required to follow more than one schedule.

The remaining paper is organized as follows: Section II elaborates the complete working of the proposed MAC protocol EEMA-MAC along with its $\mathrm{CH}$ election mechanism and RSSI estimation. Performance evaluation and results are described in Section III. Finally, concluding remarks are given in Section IV.

\section{ENERGy EFFicient Mobility Aware Mac (EEMA- MAC) PROTOCOL}

The SMAC protocol performs very well in case of stationery nodes and when the connection setup and breakup is not rapid. The mobile node has to wait for synchronization period to detect a SYNC message, if it desires to setup a connection in another. This wait time might be long and might get disconnected from the network. MS-MAC considers only for the mobility but did not propose a solution for energy efficiency. The incidence of synchronization is varied as per the node speed which lets to drain high amount of energy for speed moving vehicles. Hence we propose an algorithm which helps to expedite the connection setup and also provide energy efficiency.

The proposed protocol is an energy efficient mobility aware based MAC protocol. The basic structure of the proposed algorithm is derived from widely used SMAC protocol. It consists of periodic sleep and wakeup cycle as in S-MAC protocol with a listen and sleep period of $100 \mathrm{~ms}$. Each node periodically broadcasts a SYNC packet which consists of consists of source address, sleep time, received signal strength, speed, $\mathrm{x}$-coordinate, $\mathrm{y}$-coordinate and remaining energy of sender node. By periodically broadcasting the SYNC packet, nodes interchange their schedules to its close neighbors. The node on becoming a cluster head wake up for a longer wake up period and less sleep time than usual node in order to detect a new incoming node. On receiving a SYNC packet from the new incoming node not in the list, the $\mathrm{CH}$ quickly broadcasts its schedule in order to let the newly incoming node to synchronize with the $\mathrm{CH}$. In this way the energy of all other nodes can be conserved by waking up and sleeping at the $\mathrm{CH}$ schedule and the role of $\mathrm{CH}$ is exchanged regularly in order not to let the energy of $\mathrm{CH}$ completely drain. We introduce a cluster based approach in which a cluster is formed and each member node then synchronizes with the schedule of Cluster Head $(\mathrm{CH})$. The member nodes are scheduled to wake up at the same time as that of the cluster head. The amount of remaining energy in each node is used to choose a cluster head. The listen period is further divided into SYNC and DATA packets, respectively.

On receiving the SYNC packet each node maintains a list of immediate neighbors and is updated periodically. The cluster head election takes place after every 10 frames (10s) in order to maintain the synchronization. Clusters are formed depending on the mobility of each node. Each node calculates the relative velocity among its immediate neighbors. 


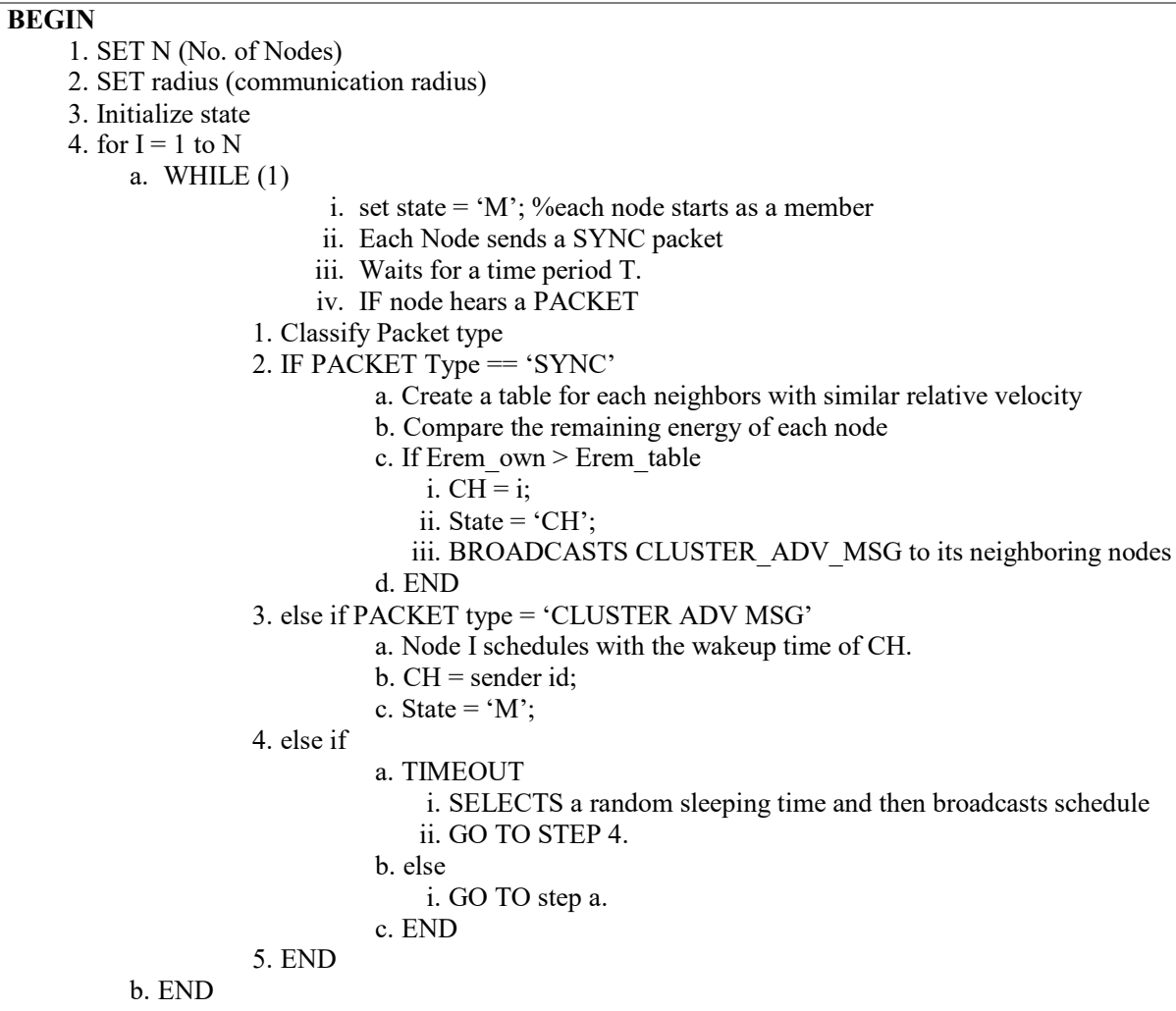

Hence the nodes with similar mobility forms a cluster and the node with highest remaining energy is elected as a cluster head, which is turn broadcasts the CLUSTER_ADV_MSG to all its immediate neighbors within its range. On receiving CLUSTER_ADV_MSG each node now schedule with the sleep time of the cluster head.

The working of EEMA- MAC protocol is explained in the form of an algorithm which can be seen in Algorithm 1 above.

\section{A. RSSI based location estimation}

Each node estimates its location depending upon the Received Signal Strength, every time it receives SYNC packet from neighboring nodes. The SYNC packet consists of the estimated $x$ and $y$ coordinate of the sender. The receiver node then calculates the angle between the sender node and itself depending upon these values. In our proposed algorithm we assume that a node requires at least 3 RSSI values to estimate the location. The path loss is assumed as

$$
p l(d)=p l\left(d_{0}\right)+10 \gamma \log _{10}\left(\frac{d}{d_{0}}\right)
$$

Where $p l$ represents path loss and $d_{o}$ is the reference distance.

$$
p l=10 * \log _{10}\left(P_{t} / P_{r}\right)
$$

Here $P_{t}$ signifies transmit power and $P_{r}$ indicates received signal strength.

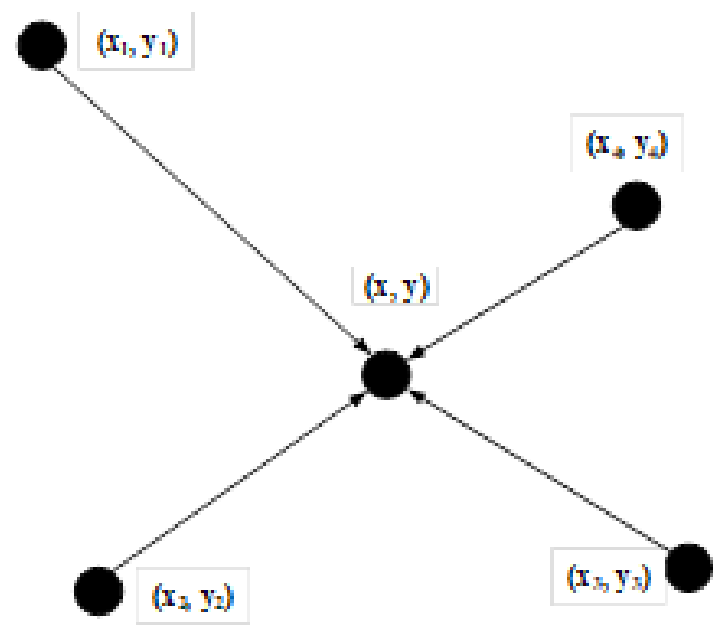

Fig. 2. Node topology.

Consider a node at location $(x, y)$ whose location is to be estimated as shown in Fig. 2. It receives a SYNC packet from $n$ neighboring nodes which consists of $\mathrm{x}$-coordinate and $\mathrm{y}$ coordinate. A target node estimates its location $(x, y)$ and calculates angle between itself and the neighboring nodes which help to find the direction of movement of each node. Let $D_{i}$ be the estimated distance between the target node and the node $\left(x_{i}, y_{i}\right)$ which is obtained using the log-model.

$$
D_{i}=\sqrt{\left(x_{i}-x\right)^{2}+\left(y_{i}-y\right)^{2}}
$$




$$
D_{i}^{2}=\left(x_{i}-x\right)^{2}+\left(y_{i}-y\right)^{2}
$$

From above equation we can write,

$$
D_{i}^{2}=x_{i}^{2}-2 x_{i} x+x^{2}+y_{i}^{2}-2 y_{i} y+y^{2}
$$

Here, $i=1,2,3,4, \ldots \ldots . n$

Similarly, for $\mathrm{n}$-th node the equation can be written as

$$
D_{n}^{2}=x_{n}^{2}-2 x_{n} x+x^{2}+y_{n}^{2}-2 y_{n} y+y^{2}
$$

From (5) and (6) it can be written as

$$
\begin{aligned}
& x_{i}^{2}+y_{i}^{2}-x_{n}^{2}-y_{n}^{2}+D_{n}^{2}-D_{i}^{2}=2 x\left(x_{i}-x_{n}\right) \\
& +2 y\left(y_{i}-y_{n}\right)
\end{aligned}
$$

$$
\begin{aligned}
& B=\left[\begin{array}{l}
x_{1}^{2}+y_{1}^{2}-x_{n}^{2}-y_{n}^{2}-D_{1}^{2}+D_{n}^{2} \\
x_{2}^{2}+y_{2}^{2}-x_{n}^{2}-y_{n}^{2}-D_{2}^{2}+D_{n}^{2} \\
\cdots \cdots \cdots \cdots \cdots \cdots \cdots \cdots \cdots \cdots \cdots \cdots \\
x_{n-1}^{2}+y_{n-1}^{2}-x_{n}^{2}-y_{n}^{2}-D_{n-1}^{2}+D_{n}^{2}
\end{array}\right]
\end{aligned}
$$

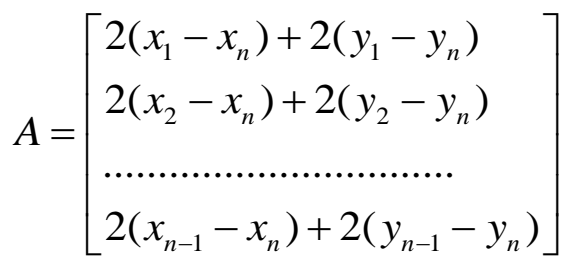

$$
\begin{aligned}
& X=\left[\begin{array}{l}
x \\
y
\end{array}\right] \\
& A X=B
\end{aligned}
$$

Three nodes are enough to locate the coordinates of unknown nodes and coordinates are calculated using trilateral localization algorithm.

After the estimation of coordinates, the angle is given by

\section{B. Cluster Head Election}

$$
\theta=\tan ^{-1}\left(\frac{y_{i}-y}{x_{i}-x}\right)
$$

When a node enters a sensing area it sends a SYNC packet and wakeup for a time period t. If the node does not listens to any schedule from its neighboring nodes, it randomly selects its sleeping time and broadcasts its schedule in a SYNC message signifying that it will go to sleep after certain time period.

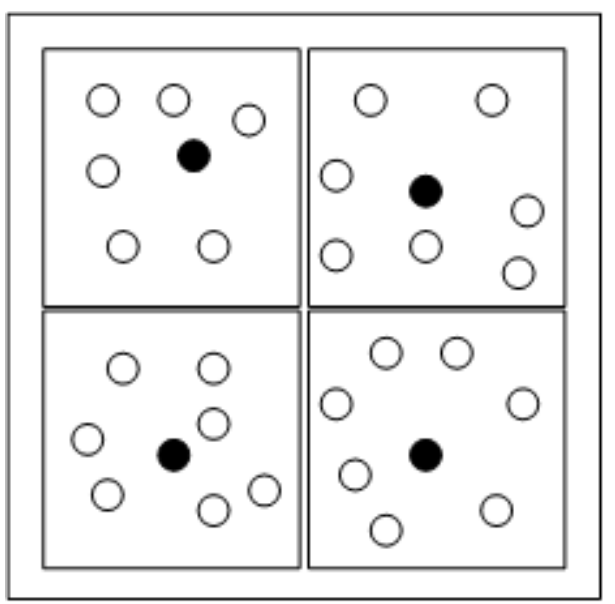

Fig. 3. Cluster formation model.

In Fig. 3, the nodes with black color are the CHs. The clustering algorithm forms a virtual cluster of nodes with similar velocity. The member nodes now synchronize with the $\mathrm{CH}$. If a node receives a CLUSTER_ADV_MSG from other $\mathrm{CH}$, it then synchronizes with schedule of the node from which it has received a broadcast message.

The cluster head election takes place after every 10 frames in which all nodes have equal probability to become a cluster head. Each node maintains a table of their neighboring nodes with similar mobility on receiving of SYNC packet. The relative velocity is now given by

$$
\Delta v=v_{A}-v_{B}
$$

Hence this helps to form a group of clusters with same mobility. Based on the information received from SYNC packet decides whether a node can become a cluster head or not. If the node has maximum energy among the group of nodes in a neighboring table, then the node immediately broadcasts CLUSTER_ADV_MSG with its own id and sleep schedule to its neighboring nodes. On hearing of this message all the neighboring nodes now synchronizes with the $\mathrm{CH}$.

For the border nodes which receive a broadcast message from more than one $\mathrm{CH}$ chooses the node which is closer to itself and then discards the other.

\section{SYNC Packet}

The format of SYNC frame is shown in Fig. 4. The member nodes have an equal sleep and wake up time as that of S-MAC as can be seen in Fig. 5 whereas the cluster head has an extended wake up time and less sleep time.

Fig. 6 shows longer wakeup time which allows the newly coming node to synchronize with the $\mathrm{CH}$. On receiving of SYNC message from a newly coming node immediately responds with an ACK packet by the $\mathrm{CH}$. Only the cluster head acknowledges with an ACK packet.

\begin{tabular}{|c|c|c|c|c|c|c|c|c|c|}
\hline T ype & L ength & $\begin{array}{c}\text { Src } \\
\text { address }\end{array}$ & $\begin{array}{c}\text { Sync } \\
\text { node }\end{array}$ & $\begin{array}{c}\text { Sleep } \\
\text { Time }\end{array}$ & Speed & $\begin{array}{c}x, y \\
\text { coordies to }\end{array}$ & $\begin{array}{c}\text { Kas aning } \\
\text { Fuergy }\end{array}$ & State & CRC \\
\hline
\end{tabular}

Fig. 4. Format of SYNC frame. 


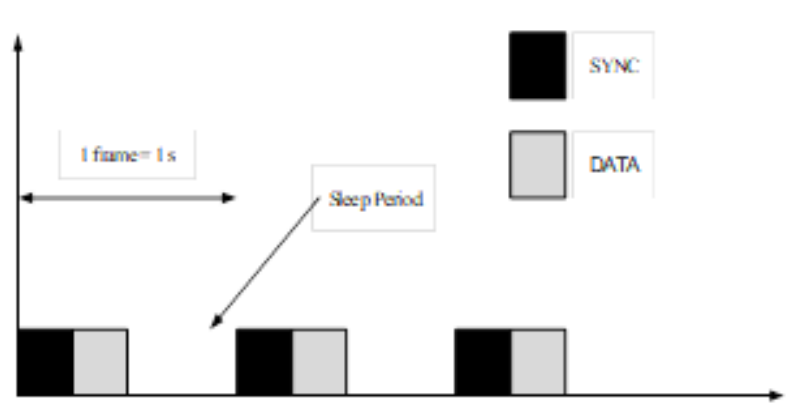

Fig. 5. Description of EEMA-MAC syncronization period.

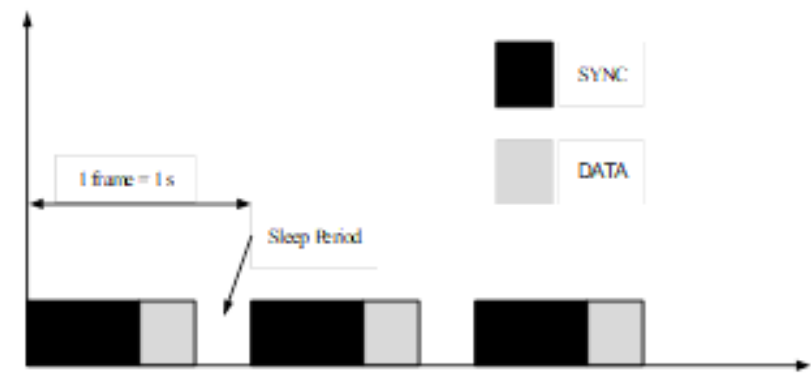

Fig. 6. Description of EEMA-MAC syncronization period for $\mathrm{CH}$.

TABLE I. SIMULATION PARAMETERS

\begin{tabular}{|l|l|}
\hline Area & {$[100 \mathrm{~m} \times 100 \mathrm{~m}]$} \\
\hline No of Nodes & 100 \\
\hline Speed & {$[1 \mathrm{~m} / \mathrm{s}-5 \mathrm{~m} / \mathrm{s}]$} \\
\hline Communication radius & $30 \mathrm{~m}$ \\
\hline Protocols & {$[$ SMAC,MS-MAC, EEMA- } \\
& MAC] \\
\hline CWmax & 100 \\
\hline Duty cycle & $10 \%$ \\
\hline Sleep Power & $0.05 \mathrm{~mW}$ \\
\hline Idle Power & $344.2 \mathrm{~mW}$ \\
\hline Transmit Power & $386 \mathrm{~mW}$ \\
\hline Receive Power & $368 \mathrm{~mW}$ \\
\hline Total Energy & $1000 \mathrm{~J}$ \\
\hline Packet Size & 50 bytes \\
\hline
\end{tabular}

\section{PERFORMANCE EVALUATIONS}

EEMA-MAC is compared with Sensor-Medium Access Control (S-MAC) and Mobility-aware Sensor MAC (MSMAC) protocols in order to evaluate its performance and efficiency. Simulation results conclude that EEMA-MAC enhances the overall throughput. Moreover it decreases energy consumption and packet loss compared with existing protocols.

\section{A. Simulation Parameters}

Nodes are deployed randomly over $100 \mathrm{~m} \times 100 \mathrm{~m}$ region. Number of nodes for this simulation is fixed to $100(n=100)$. Fig. 4 shows the randomly deployed nodes for our evaluations.

The parameters given in Table I are used to simulate $\mathrm{S}$ MAC [1], MS-MAC [9] and EEMA-MAC.

\section{B. Simulation Results}

To achieve the desired results, MATLAB is used as a simulation tool. Firstly, we need to analyze the energy consumption of all the protocols. To calculate the average energy consumption we use the formula:

$$
\text { Average_Energy_Consumption }_{=}=\frac{\sum_{i=1}^{N} E-E_{r e m}}{N}
$$

The speed of the nodes is varied from $1 \mathrm{~m} / \mathrm{sec}$ to $5 \mathrm{~m} / \mathrm{sec}$. Fig. 7 shows that with increase of speed, the average energy consumption also increases. The energy consumption of EEMA-MAC protocol has less energy consumption as compared to other protocols. The energy consumption is highest for SMAC protocol which shows that it is highly inefficient in mobile environments since the nodes consume more energy in maintaining the schedules.

Secondly, we have to calculate the throughput which is termed as the total number of packets transferred over total simulation time. With the increase of speed, the throughput decreases. Since the increase in speed causes less packets to reach to the destination. The throughput for EEMA-MAC is the highest whereas the SMAC protocol shows lowest throughput as shown in Fig. 8.

With the increase of speed, quantity of dropped packets also increases. Thus, performance at a node cannot be just measured in terms of delay, but also in terms of probability of the packet being dropped. In order to make sure that all the data is eventually transported from source to destination, a retransmission of dropped packet can be done on end to end basis. Also, the losses between $5 \%$ and $10 \%$ of the total packet stream will disturb the network performance significantly. The higher is the speed, the higher is the number of losses. The packet drop is seen to be much less in EEMA-MAC protocol then SMAC and MS-MAC protocol as can be seen in Fig. 9. We can calculate the Packet drop by using the formula given below:

$$
P D(\%)=\frac{\text { Total.no.of } . \text { packets.sent }- \text { Total } \text {.no.of } \cdot \text { packets.received }}{\text { Total.no.of.packets.sent }} \times 100
$$

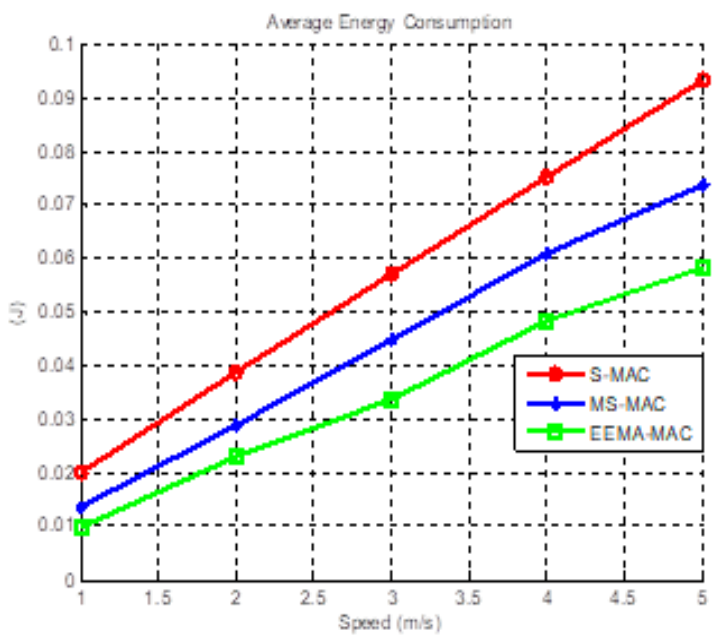

Fig. 7. Average energy consumption. 


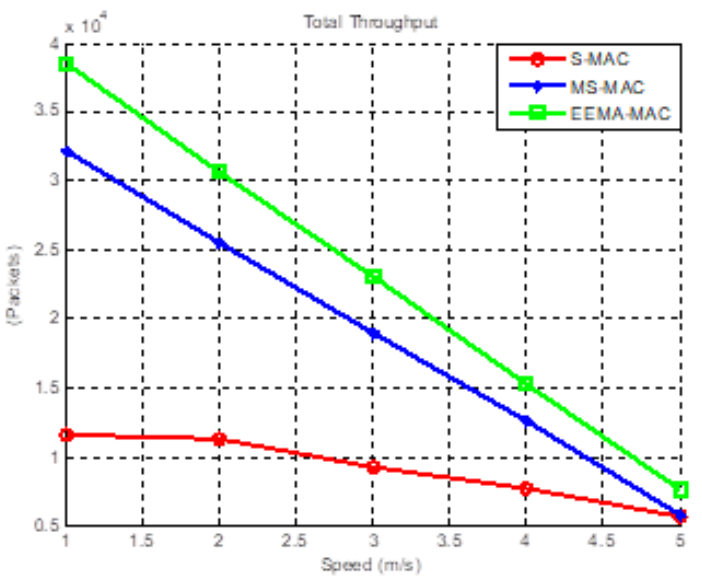

Fig. 8. Total throughput.

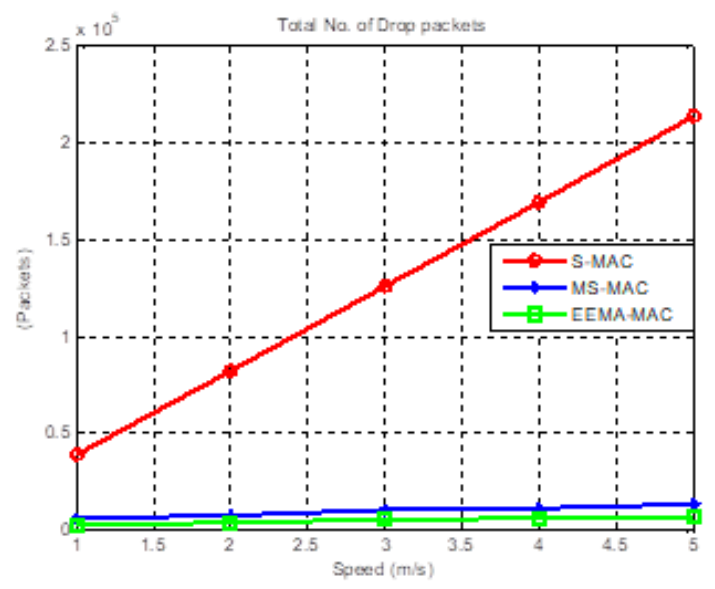

Fig. 9. Total number of drop packets.

\section{CONCLUSION}

A number of potential applications (areas) are on the rise in the field of Wireless Sensor Network. The energy efficient MAC protocols turn out to be enormously significant and are indeed subject to many present research projects. In our current research, we have proposed an energy efficient MAC protocol called EEMA-MAC for WSNs. The major aim is to increase the efficiency of the network by extending the wake up time and reducing the sleep time at $\mathrm{CH}$ end. Performance evaluation and simulation results conclude that EEMA-MAC has made an significant improvement in terms of energy consumption, throughput and packet loss in comparison with previously proposed MAC protocols such as S-MAC and MS-MAC. In the future, we can further evaluate the effectiveness of our proposed protocol by varying the number of nodes and can conduct more simulations involving random topologies.

\section{REFERENCES}

[1] W. Ye, J. Heidemann, and D. Estrin, "An energy-efficient mac protocol for wireless sensor networks," IEEE Twenty-First Annual Joint Conference of the IEEE Computer and Communications Societies. Proceedings, vol. 3, pp. 1567 - 1576, 2002.

[2] F. Peng, and M. Cui, "An Energy-Efficient Mobility-Supporting MAC protocol in Wireless Sensor Networks," Journal of Communications and Networks, vol. 17, no. 2, 2015.

[3] F. Peng, "A novel Adaptive Mobility-Aware MAC protocol in Wireless Sensor Networks," Springer Wireless Personal Communications Journal, vol. 81, no. 2, pp. 489-50, 2015.

[4] W. Dargie and C. Poellabauer, Fundamentals of Wireless Sensor Networks: Theory and Practice. Wiley Publishing, 2010.

[5] A. Mainwaring, D. Culler, J. Polastre, R. Szewczyk, and J. Anderson, "Wireless sensor networks for habitat monitoring," in ACM International Workshop on Wireless Sensor Networks and Applications (WSNA 2002), 2002, pp. 88-97.

[6] S. Kim, S. Pakzad, D. Culler, J. Demmel, G. Fenves, S. Glaser, and M. Turon, "Health monitoring of civil infrastructures using wireless sensor networks," in IPSN '07: Proc. 6th international conference on Information processing in sensor networks. New York, NY, USA: ACM, 2007, pp. 254-263.

[7] G. Werner-Allen, K. Lorincz, M. Welsh, O. Marcillo, J. Johnson, M. Ruiz, and J. Lees, "Deploying a wireless sensor network on an active volcano," IEEE Internet Computing, vol. 10, no. 2, pp. 18-25, 2006.

[8] I. Stoianov, L. Nachman, S. Madden, and T. Tokmouline, "PIPENET: a wireless sensor network for pipeline monitoring," in IPSN '07: Proc. 6th international conference on Information processing in sensor networks. New York, NY, USA: ACM, 2007, pp. 264-273.

[9] H. Pham and S. Jha, "An adaptive mobility-aware mac protocol for sensor networks (ms-mac)," IEEE International Conference on Mobile Ad-hoc and Sensor Systems, pp. 558 - 560, October 2004.

[10] S. W. A. Kazmi, A. Kacso, and R. Wismuller, "Recent MAC Protocols for Mobility-Aware Wireless Sensor Networks - A Survey and Future Directions," IEEE Ninth International Conference on Ubiquitous and Future Networks (ICUFN), July 2017.

[11] H. R. Silva, J. S. Silva, and F. Boavida, "Mobility in wireless sensor networks-survey and proposal," Computer Communications, vol. 52, pp. 1-20, 2014.

[12] S. W. A. Kazmi, A. Kacso, and R. Wismüller, "On MAC design for mobility-aware wireless sensor networks," IEEE 2nd International Conference on Computer and Communication Systems (ICCCS), pp. 152-157, July 2017. 